\title{
Highly sensitive electrochemical sensor for the detection of Shiga toxin-producing $E$. coli (STEC) using interdigitated micro-electrodes selectively modified with a chitosan-gold nanocomposite
}

\author{
Luiza A. Wasiewska ${ }^{\mathrm{a}, \mathrm{b}}$; Fernando Garrido Diaz ${ }^{\mathrm{a}}$, Han Shao ${ }^{\mathrm{a}}$, Catherine M. Burgess ${ }^{\mathrm{b}}$, Geraldine Duffy $^{\mathrm{b}}$, Alan O’Riordan*a \\ ${ }^{a}$ Nanotechnology group, Tyndall National Institute - University College Cork, Dyke Parade, Cork, Ireland T12 R5CP \\ ${ }^{\mathrm{b}}$ Food Safety Department, Teagasc Food Research Centre, Ashtown, Dublin, Ireland D15 DY05
}

KEYWORDS: DNA, bacteria detection, methylene blue, square wave voltammetry, silicon chips, electrochemical detection, genosensor

\begin{abstract}
Shiga toxin-producing E. coli (STEC) is a foodborne pathogen of great concern due to the severity of the disease it can cause. A key pathogenicity factor is the ability to produce Shiga T Toxin 1 and 2, which are encoded by genes stx. Herein we report the development of a highly sensitive, label-free, electrochemical DNA-based sensor for detection of stxl gene using interdigitated gold microelectrodes (IDEs) on fully integrated silicon chips. Each IDE comprised a working IDE, used for DNA probe immobilisation and an accumulator IDE. The working IDE was modified with gold nanoparticles (Au NPs) and chitosan gold nanocomposite to allow a covalent attachment of amine-modified probe DNA. The electrochemical detection was undertaken using methylene blue $(\mathrm{MB})$ as a redox molecule, which intercalated into the double-strand DNA. The accumulator IDE was used for the electrostatic accumulation of the MB to the DNA binding region of the sensor thereby greatly enhancing sensitivity. Reduction of MB was recorded using square wave voltammetry (SWV). Using this approach, we achieved a linear response between $10^{-16}$ and $10^{-6} \mathrm{M}$ of synthetic target strand with the lowest measured limit of detection of $100 \mathrm{aM}$ after 20 minutes hybridisation time. Subsequently, chromosomal DNA from four different $E$. coli strains (two stxl positives and two stxl negatives), Listeria monocytogenes and Bacillus cereus was used to confirm the selectivity of the presented method. This novel on-chip biosensor for the detection of STEC has the potential to be used in point-of-use detection.
\end{abstract}

\section{Introduction}

Shiga toxin-producing E. coli (STEC) is a food-borne pathogen of a great public health concern, which can cause severe illness including haemolytic uremic syndrome (HUS), that may even lead to death [1]. Cattle are known to be their main reservoir, and the most typical source of infection is via the consumption of contaminated undercooked beef meat $[2,3]$. Since most E. coli are harmless and a part of healthy gut flora in humans and animals, specific detection of STEC is crucial for preventing infections. Since the big multinational STEC O104 outbreak in Germany in 2011, plus an increasingly wide range of STEC serogroups linked to human illness, the targets for detection has shifted off from serogroup based approaches towards targeting the genes coding for Shiga toxin production (stxl or stx2); which are major virulence factors of STEC $[4,5]$. The foremost common technique for DNA based detection is polymerase chain reaction (PCR), in which the specific target sequence from the DNA extracted from the organism is amplified, significantly increasing the sensitivity of the method. Especially real-time PCR (qPCR) found a large application in pathogens during which the number of amplified DNA at the particular time is monitored using fluorescent probes [6]. This approach offers significant advantages like high sensitivity and selectivity, however, it is time-consuming and requires highly trained staff to perform experiments, making it unsuitable for point-of-use detection $[7,8]$. A potential alternative is to employ solid-state electrochemical DNA biosensors, comprising a probe DNA as a recognition element and a transducer, which provides a measurable signal following a DNA hybridisation event [9]. Their advantages over traditional techniques include the speed in obtaining results, simplicity of use, and suitability for miniaturisation and portability [10]. The key aspects to consider when developing DNA based electrochemical biosensors are (i) sensor miniaturisation [11] (ii) requirements for robust probe attachment [12], and (iii) detection methods with low complexity [13], all of which have enormous impacts on the ultimate performance and use. Miniaturised electrochemical sensors $(<10 \mu \mathrm{m}$ diameter) benefit from enhanced analyte mass transport, reduced background noise, and high current densities making them an ideal choice for point-of-use sensors.

Interdigitated microelectrodes (IDEs), comprising two comb-like closely spaced electrodes which are individually electrically connected, have found several applications in point-of-use sensing [14]. For instance, they have recently been applied for the detection of a variety of heavy metals species in water where one comb was used as a working electrode and the other as a generator electrode, allowing electrochemical modification of local $\mathrm{pH}$ for improving sensing efficacy $[15,16]$. They have also been applied to DNA detection where the probe DNA was either immobilised on both IDEs [17] or the silicon surface between them [18-20]. Typically, the DNA hybridisation was detected using conductance measured between the two IDEs or impedance, when IDEs were used. Selective modification of only one IDE could enable using the other IDE for other function that sensing as well as using other techniques for DNA detection, such as linear sweep voltammetry. It is a straightforward electrochemical technique that has been applied for DNA detection using redox-active molecules, such as methylene blue (MB) [21], anthraqinone-2-sulfonic acid monohydrate sodium salt (AQMS) [22] or daunomycin (DNR) [23]. These molecules have different interaction mechanisms to single-stranded (ssDNA) and double-strand DNA (dsDNA). The advantages of such an approach include the short time needed to obtain results and the simplicity of the system.

The attachment of probe DNA in a stable and robust manner remains a key challenge in electrochemical sensor development [12]. 
In this regard, chitosan, a natural polymer, has gained a lot of attention as it is rich in amine groups, thereby allowing subsequent covalent attachment of a range of biomolecules [24]. It has been widely used in the development of DNA based biosensors, where typically the probe DNA modified with an amine group is attached to the polymer using glutaraldehyde as a linker [21,25]. Since it is a non-conductive polymer, several researchers have combined it with other conductive 0D, 1D, and 2D materials [26], [27], [28], [29] to enhance the electrical conductivity and therefore the sensitivity of detection. However, these modifications can be complex, e.g., require several steps, high temperatures, and thus increase the complexity of sensor development [30, 31]. In contrast, Du, Luo [32], developed a simple, one-step co-electrodeposition technique for chitosan with simultaneous deposition of gold nanoparticles on large $2 \mathrm{~mm}$ diameter electrodes. Their technique was applied for glucose detection and no biomolecule attachment was tested in their work.

In this work, gold IDEs fabricated on silicon chips were used for the development of a voltammetric sensor allowing highly sensitive detection of the STEC virulence gene, stxl. The sensor IDE was specifically modified with Cht-Au nanocomposite and probe DNA leaving the other IDE (accumulator IDE) unmodified, confirmed with optical and fluorescent microscopy. This accumulator IDE was then used to pre-concentrate a redox probe, MB, around the sensor IDE by applying open circuit potential (OCP), enhancing the hybridisation signal and increasing sensitivity. To the best of our knowledge, interdigitated electrodes have never been utilised to enhance molecule accumulation for DNA detection. By this approach, we have managed to achieve highly sensitive detection of $100 \mathrm{aM}$ and detection of chromosomal DNA without the amplification step. This non-complex method of electrode modification and DNA detection has the potential to be applied to multiplex detection of different DNA strands in the future.

\section{Materials and methods}

2.1. Chemicals

Chitosan, $\mathrm{HAuCl}_{4}, \mathrm{~N}, \mathrm{~N}-$ Dimethylformamide (DMF), KCL, PBS, sodium acetate, $50 \%$ glutaraldehyde, atto565 NHS ester, HEPES, were obtained from Sigma Aldrich, Ireland. All solutions were prepared by diluting with ultra-pure Milli-Q water $(18.2 \mathrm{M} \Omega . \mathrm{cm}$, Milli-Q). All synthetic oligonucleotides were obtained from Sigma Aldrich Ireland in a dried form. Upon arrival they were diluted with sterile DI water to $100 \mu \mathrm{M}$ and stored at $-20^{\circ} \mathrm{C}$. Before use, they were diluted in $0.1 \mathrm{M}$ PBS buffer to the desired concentration. The probe sequence for stxl detection was selected based on ISO/TS 13136:2012 standard. The probe was modified with an amine group at the 5' end, enabling covalent attachment to the electrode. For fluorescent confirmation, the target sequence was modified on the 5 ' end with Atto565 fluorescent dye. The sequences used in this work are detailed in Table 1 .

\section{Table 1. Summary of DNA sequences used in this work}

\begin{tabular}{|l|l|}
\hline Name & Sequence \\
\hline stx1 probe & $\begin{array}{l}\text { 5' NH2 (C6) CTG GAT GAT CTC AGT } \\
\text { GGG CGT TCT TAT GTAA 3' }\end{array}$ \\
\hline stx1 target & $\begin{array}{l}\text { 5' TTAC ATA AGA ACG CCC ACT GAG } \\
\text { ATC ATC CAG 3' }\end{array}$ \\
\hline $\begin{array}{l}\text { stx1 target } \\
+ \text { Atto565 }\end{array}$ & $\begin{array}{l}\text { 5' [Atto 565] TTAC ATA AGA ACG CCC } \\
\text { ACT GAG ATC ATC CAG 3' }\end{array}$ \\
\hline Non-target & $\begin{array}{l}\text { 5' CCGA TGC TAC GTC AAT GTA ACT } \\
\text { GAT TGA GCT 3' }\end{array}$ \\
\hline $\begin{array}{l}\text { Non-target } \\
+ \text { Atto565 }\end{array}$ & $\begin{array}{l}\text { 5' [Atto 565] GGA GCA GTT TCA GAC } \\
\text { AGT GCC TGA CGA 3' }\end{array}$ \\
\hline
\end{tabular}

Chitosan stock solution $(0.2 \%)$ was prepared by dissolving $0.1 \mathrm{~g}$ of chitosan in $50 \mathrm{~mL}$ DI water containing $1 \%$ acetic acid. The solution was stirred overnight and filtered using filter paper to remove undissolved polymer. The final $\mathrm{pH}$ of the stock solution was $\sim 3$. Two gold ions stock solutions were prepared and stored at $4^{\circ} \mathrm{C}$. The first stock solution, used for AuNPs electrodeposition, contained 1000 ppm $\mathrm{HAuCl}_{4}$ dissolved in $0.01 \mathrm{M}$ sodium acetate, $\mathrm{pH} 3$, while the other stock solution, used for Cht-Au nanocomposite electrodeposition, contained $1000 \mathrm{ppm}$ of $\mathrm{HAuCl}_{4}$ dissolved in DI water.

\subsection{Apparatus}

All voltametric measurements were undertaken using a CHI920 potentiostat while electrochemical impedance measurements (EIS) were undertaken using a Multi AutoLab M101. A three-electrode electrochemical setup was used for modification of one comb of a gold IDE (used as the working electrode), an on-chip gold counter electrode and an external $\mathrm{Ag} / \mathrm{AgCl}$ reference electrode. A modified four-electrode setup was employed for DNA detection: a modified gold IDE was used as a sensor, an unmodified gold IDE was used as an accumulator and on-chip gold and platinum as counter and pseudo reference electrodes, respectively.

White light optical microscopy images and fluorescent microscopy images of the non-modified and modified IDEs were acquired using an Axioskop II (Carl Zeiss Ltd.) microscope equipped with a halogen lamp and a charge-coupled detector camera (CCD; Coolsnap CF, Photometrics). The surface morphology and compositional analysis of AuNPs and Cht-Au modified IDEs were performed using a field emission scanning electron microscope (FEI QUANTA 650 HRSEM) with energy-dispersive X-ray spectroscopy (EDX Oxford Instruments INCA energy system).

\subsection{Chips fabrication}

IDEs with $10 \mu \mathrm{m}$ gaps were fabricated on silicon chip substrates. Each silicon chip comprised six sensors containing two IDEs, a gold counter electrode, and a platinum pseudo-reference electrode. Gold contact pads and interconnection metallisation on two sides of the chip allowed electrical connection to both IDEs. The method of chip fabrication was described previously [33, 34]. In brief, fourinch silicon wafer substrates bearing a $\sim 300 \mathrm{~nm}$ layer of thermally grown silicon dioxide were used. IDEs were first fabricated using optical lithography, metal evaporation (Ti $10 \mathrm{~nm} / \mathrm{Au} 150 \mathrm{~nm}$ Temescal FC-2000 E-beam evaporator) and lift-off techniques to yield well-defined, stacked metallic (Ti/Au) microband $(1 \mu \mathrm{m}$ width, $50 \mathrm{~nm}$ height, $45 \mu \mathrm{m}$ length) structures. Each chip comprised six independent sensors. A second optical lithographic and metal deposition ( $\mathrm{Ti} 10 \mathrm{~nm} / \mathrm{Ni} 70 \mathrm{~nm} / \mathrm{Au} 200 \mathrm{~nm}$ ) process was then undertaken to define a MicroSD pin-out, interconnection tracks, as well as counter electrodes ( $500 \mu \mathrm{m}$ wide $\mathrm{x} 10 \mathrm{~mm}$ long). Finally, a passivation $\mathrm{SiN}_{\mathrm{X}}$ layer was deposited on the chip by PECVD, with windows opened in this layer directly above the working, reference and counter electrodes. The windows defined the length of the working electrode to be $45 \mu \mathrm{m}$. In this work, an on-chip microSD style electrical pin-out was included to permit a facile electrical connection to external electronics. In this manner, chips could be easily swapped in and out with the potentiostat, enabling rapid analysis of multiple samples. A custom-built cell was designed and fabricated so that when screwed together, the microSD primary contact pads protruded out of the holder to allow connection with a PCB mounted microSD port.

\subsection{DNA sensor development}

Prior to electrode modification, silicon chip was cleaned by sonicating in ethanol and DI water, for ten minutes each. Once clean, the chip was dried in a stream of nitrogen, placed in a chip holder and connected to the potentiostat using either an SD connector or probe pins. Each sensor IDE was modified with gold nanoparticles, electrodeposited by applying $-0.2 \mathrm{~V}$ for $60 \mathrm{~s}$ in $500 \mu \mathrm{L}$ of $400 \mathrm{ppm}$ $\mathrm{HAuCl}_{4}$ dissolved in $10 \mathrm{mM}$ sodium acetate, $\mathrm{pH} 3$. Subsequently, $\mathrm{Cht}-\mathrm{Au}$ nano-complex was electrodeposited on top of the preAuNPs modified IDE. The solution was prepared by diluting chitosan from a stock solution $(0.2 \%)$ to $0.04 \%(\mathrm{v} / \mathrm{v})$ with DI water 
containing $1 \%$ acetic acid. Afterwards, $1 \mathrm{M} \mathrm{NaOH}$ was added to the solution to increase its final $\mathrm{pH}$ to $\sim \mathrm{pH} 5$. Gold ions from a (DI water) stock solution were added to chitosan just before the deposition to achieve a final concentration of $50 \mathrm{ppm} \mathrm{HAuCl}_{4}$. The Cht$\mathrm{Au}$ solution was mixed using a vortex and $500 \mu \mathrm{L}$ was added to the sample well. A voltage of $-1.5 \mathrm{~V}$ was applied for 15 seconds to each electrode. Subsequently, the modified chip was immersed in 0.01 $\mathrm{M}$ PBS buffer, $\mathrm{pH}$ 7, for 10 minutes to increase the stability of the layer, washed thoroughly with DI water and dried in a stream of nitrogen. The electrodes were then immersed in $0.2 \%(\mathrm{v} / \mathrm{v})$ glutaraldehyde for 2 hours. Afterwards, a $50 \mu \mathrm{L}$ droplet of $0.5 \mu \mathrm{M}$ aminemodified probe ssDNA was deposited on top of an electrode, incubated for 2 hours, then the unattached probe was removed with DI water.

\subsection{Fluorescence characterisation}

To confirm a uniform electrodeposited chitosan layer, modified electrodes were immersed in a fluorescently labelled succinimidyl ester (Atto565-NHS ester) which is known to bind to the primary amine groups. First, $0.5 \mathrm{mg}$ Atto565-NHS ester was reconstituted in $500 \mathrm{uL}$ DMF. Then, $50 \mu \mathrm{L}$ of this solution was diluted in $30 \mathrm{~mL}$ of PBS buffer. Chips with chitosan modified electrodes were immersed in this mixture and incubated for 30 minutes at room temperature. After this time, chips were removed, washed with DI water to remove the unattached dye and dried in a stream of nitrogen. Fluorescent microscopy was then used to confirm that the target DNA hybridised with the probe DNA and that no unspecific binding took place. Two different DNA strands, target and non-target, tagged with Atto565 were employed. AuNPs/CHI-Au/ssDNA modified sensor IDEs were incubated for 30 minutes with either 10 nM target DNA or non-target DNA both DNA strands were tagged with Atto565. Afterwards, chips were washed with DI water, dried under nitrogen. and characterised using fluorescent microscopy.

2.6. Hybridisation detection and methylene blue accumulation using open circuit potential

Square wave voltammetry (SWV) using methylene blue as a redox molecule was employed for hybridisation detection. Methylene blue is well known to interact with single and double-strand DNA based using different modes of action. After hybridisation, a chip was immersed in $50 \mu \mathrm{M}$ methylene blue in HEPES buffer $(20 \mathrm{mM}$ HEPES, $10 \mathrm{mM} \mathrm{KCl}, \mathrm{pH}$ 7) for 10 minutes, with an applied OCP for the first five minutes to accumulate the MB. After this time, MB was washed away with HEPES buffer followed by SWV recorded between 0 and $-0.8 \mathrm{~V}$ versus on the platinum pseudo reference electrode (frequency $75 \mathrm{~Hz}$, pulse amplitude $75 \mathrm{mV}$, increment 15 $\mathrm{mV})$.

\subsection{Culture preparation and target DNA extraction}

DNA was extracted from four E. coli strains, Listeria monocytogenes and Bacillus cereus. Two E. coli strains contained the stxl gene (O157 - ATCC35150 and O103) while the other two strains did not contain this gene (O157 - NCTC12900 and O91 09_A_15_1_1). Briefly, the cultures were grown up from culture collection stocks (Teagasc Food Research Centre Ashtown) which were stored on protective beads at $-80^{\circ} \mathrm{C}$. A single bead of each isolate was streaked on Tryptone Soy Agar (Oxoid, Fisher Scientific Ireland) and incubated overnight at $37{ }^{\circ} \mathrm{C}$. An isolated colony was then placed in Tryptone Soy Broth and incubated overnight at $37^{\circ} \mathrm{C}$. DNA was extracted from the overnight culture using a Qiagen DNeasy Blood and Tissue kit (Qiagen, Manchester, UK), and in accordance with the manufacturer's instructions. The DNA concentration was measured using a Qubit dsDNA BR Assay Kit (Thermo Fisher Scientific, Ireland) on a Qubit 4.0 fluorometer.

Before electrochemical measurements, DNA samples were diluted 5 times in $0.1 \mathrm{M}$ PBS buffer, $\mathrm{pH} 7.2$, and heated to $95{ }^{\circ} \mathrm{C}$ for 5 minutes to denature dsDNA. Subsequently, the samples were cooled down on ice and incubated on prepared sensors for 30 minutes at room temperature followed by electrochemical measurements.

\section{Results and discussion}

\subsection{Characterisation of modified electrodes}

Figure 1. (A) presents a picture of the silicon chip. In this work, an external $\mathrm{Ag} / \mathrm{AgCl}$ reference electrode was used during the deposition of AuNPs and Cht-Au nanocomposite, while the on-chip pseudo-reference electrode was used for DNA detection. Figure 1. (B) shows a silicon chip in a chip holder. Chitosan was electrodeposited at sensor IDE in presence of Au ions, similar to Du, Luo [32]. To find the optimal potential for cathodic deposition of chitosan, cyclic voltammetry (CV) in Cht-Au solution $(0.04 \%$ chitosan, $50 \mathrm{ppm} \mathrm{Au}$ ) at $\mathrm{pH} \sim 5$ was undertaken. The potential was cycled between $0 \mathrm{~V}$ and $-2 \mathrm{~V}$, see Figure 1. (C), and it was found that the cathodic current increased significantly around $-1.4 \mathrm{~V}$ suggesting the commencement of hydrogen evolution and therefore an increase in $\mathrm{pH}$ around the electrode. The observed current remained stable (plateaued) until -1.8 V whereon it started increasing rapidly at more cathodic applied voltages. Consequently, based on CV data, the potential range of interest for Cht-Au deposition was found to be between -1.4 and $-1.8 \mathrm{~V}$. To confirm this, multiple electrodepositions were undertaken in the potential of $-1.2 \mathrm{~V}$, and $-2 \mathrm{~V}$ and visualised under optical microscopy. Figure S1. shows that a defined polymer layer was electrodeposited at sensor IDEs for potentials of $-1.4,-1.5$ and -1.6 , which supports the results based on $\mathrm{CV}$. At $-1.2 \mathrm{~V}$ the $\mathrm{pH}$ change was not sufficient for chitosan deposition at the electrode while at greater than $-1.8 \mathrm{~V}$ the hydrogen evolution was so rapid that the polymer deposited around the electrode instead of specifically at an array. Following these experiments, a potential of $-1.5 \mathrm{~V}$ was selected as optimal for chitosan deposition.

$\mathrm{CV}$ in $5 \mathrm{mM} \mathrm{Fe}{ }^{4-} / \mathrm{Fe}^{3-}$ in $0.1 \mathrm{M} \mathrm{KCl}$ as supporting electrolyte (scan rate $100 \mathrm{mV} / \mathrm{s}$ ) were undertaken to characterise the different gold surfaces of a pristine IDE and post modified IDEs. In addition, the electrodeposition of a gold nanoparticle layer onto gold electrodes was explored to increase surface roughness prior to Cht-Au deposition. Figure 1. (D) presents CV for (i) unmodified gold, (ii) Cht$\mathrm{Au}$, (iii) AuNPs, and (iv) AuNPs/Cht-Au. For pristine gold electrodes, a well-defined voltammogram exhibited typical characteristics for the $\mathrm{Fe}^{4-} / \mathrm{Fe}^{3-}$ redox couple with a peak oxidation current of $\sim 25 \mathrm{nA}$ and low hysteresis was observed. Following electrodeposition of a Cht-Au complex at the unmodified electrode, a significant decrease in the magnitude of the redox currents for the $\mathrm{Fe}^{-}$ ${ }^{4} / \mathrm{Fe}^{3-}$ redox couple was observed $(\sim 3 \mathrm{nA})$. This suggests a Cht-Au layer deposited effectively onto the electrode surface and that this layer had a lower conductivity and thus partially insulated the surface of the electrode; due to the lack of conduction pathways from the underlying electrode to the modified layer. We attribute the lack of these pathways to the smoothness of the evaporated gold of the electrode. An increase in $\mathrm{Fe}^{-4} / \mathrm{Fe}^{3-}$ current magnitudes, compared to the pristine gold electrodes $(\sim 50 \mathrm{nA})$, were observed for IDE electrodes first modified with an Au-NP layer, which may be attributed to an increase in surface area. A Cht-Au layer was then deposited onto these modified electrodes and characterised by $\mathrm{CV}$ and electrochemical impedance spectroscopy (EIS). From the CV data, it was observed that (unlike the unmodified electrodes) the addition of the Cht-Au layer, resulted in a slight increase in the $\mathrm{Fe}$ ${ }^{4} / \mathrm{Fe}^{3-}$ current magnitudes and also decreased the separation between the oxidation and reduction peaks, suggesting a successful deposition of this layer. The CV results are supported by the EIS data presented in Figure 1 (E) where a decrease in impedance was observed from $12.2 \mathrm{M} \Omega$ at unmodified IDE to $5 \mathrm{M} \Omega$ following modification of the underlying AuNPs layer, due to the increased surface area and 5.5 M $\Omega$ following deposition of a subsequent Cht$\mathrm{Au}$ layer. 

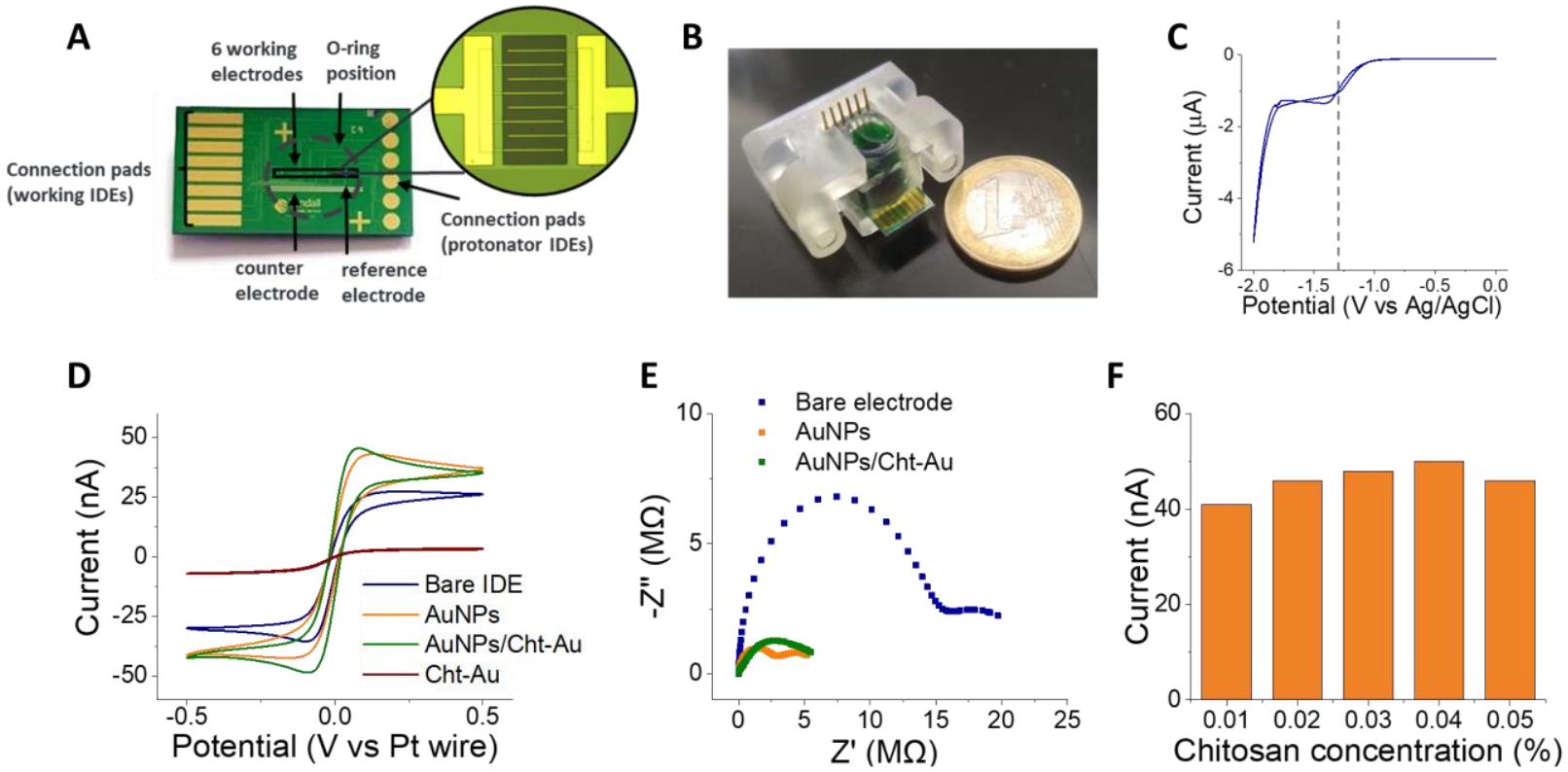

Figure 1. (A) Picture of a fully integrated silicon chip and an optical image of IDE. (B) Silicon chip in a chip holder. (C) Cyclic voltammogram at the unmodified sensor IDE in $0.04 \%$ chitosan, $50 \mathrm{ppm} \mathrm{Au} \mathrm{pH} \mathrm{5.} \mathrm{(D)} \mathrm{Cyclic} \mathrm{voltammograms} \mathrm{in} 5 \mathrm{mM} \mathrm{Fe} 4-/ \mathrm{Fe} 3-, 0.1 \mathrm{M} \mathrm{KCl}$ at (i) unmodified IDE and after deposition of (ii) $0.4 \%$ chitosan with 50 ppm HAuCl4 (Cht-Au), (iii) AuNPs, and (iv) AuNPs/Cht-Au. (E) Impedance measurements in $5 \mathrm{mM} \mathrm{Fe} 4-/ \mathrm{Fe} 3-, 0.1 \mathrm{M} \mathrm{KCl}$ at (i) unmodified IDE and after deposition of (ii) AuNPs and (iii) AuNPs/Cht-Au. (G) Oxidation peak current of $\mathrm{CV}$ in $5 \mathrm{mM} \mathrm{Fe}{ }^{4-} / \mathrm{Fe}^{3-}, 0.1 \mathrm{M} \mathrm{KCl}$ at IDE modified with ChtAu nanocomposite where the concentration of CHI varied between $0.01 \%$ and $0.05 \%$.

The effect of varying the Cht concentrations within the Cht-Au complex was also investigated at IDEs pre-modified with AuNPs, see Figure $1(\mathrm{~F})$. Cht-Au complexes were deposited at $-1.5 \mathrm{~V}$ for 15 seconds with the $\mathrm{CHI}$ concentrations varied between $0.01 \%$ and $0.05 \%$. It was found that the conductivity of the electrode increased with the increasing chitosan concentration reaching a maximum at $0.04 \%$ of chitosan and decreasing thereafter. This suggests that, with increasing chitosan concentration in a solution, the Cht enables the formation of more gold nanoparticles at an electrode, which results in an increase in electrode conductivity. However, at higher Cht concentrations, the Cht: Au ratio becomes unfavourable, thereby decreasing the conductivity of the electrode. Figure S2. shows the electrochemical characterisation of AuNPs/Cht-Au IDE in $5 \mathrm{mM} \mathrm{Fe} e^{-4} / \mathrm{Fe}^{3-}$ redox couple at different scan rates $(10 \mathrm{mV}-200$ $\mathrm{mV}$ ). As expected, the peak currents increased with an increased scan rate, suggesting that the redox reaction is diffusion-controlled $[35,36]$. The inset shows linear relationships of the anodic and cathodic peak currents as a function of the square root of scan rate which indicates that the diffusional profiles of individual micro bands overlap and the electrode behaves like a larger microelectrode.

Following electrode optimisation as described above, an optical and electron microscopy characterisation study was undertaken in Figure 2. and Figure S3. Figure 2 (A) shows an optical image of a sensor following modification of one IDE (dark bands) with AuNPs. It can be seen that the modification only occurred at a sensor IDE while the accumulator IDE remained unmodified. Figure 2. (B) shows an SEM image of the AuNPs modified sensor IDE and an inset at higher magnification. Two different nanostructure morphologies were observed, round nanoparticles on the length of the electrode and bigger clusters at the electrode edges. The size of the round NPs varied between $\sim 30 \mathrm{~nm}$ and $\sim 90 \mathrm{~nm}$ (see Figure S3. (A)) while larger dendritic particles, $\sim 500 \mathrm{~nm}$, were observed at the band edges. These larger particles arose from the high electric fields, present at the boundary discontinuities occurring at the interface between the top surface and sidewalls of the bands, enhancing gold deposition. Figure 2. (C) shows an optical image of a microelectrode, with a sensor IDE following AuNPs/CHI-Au modification. A defined green coloured layer can be seen around each band in the sensor IDE, compared to the electrode modified with just AuNPs only (Figure 2. (A)). This green layer is attributed to the chitosan and suggests that the polymer was deposited on top of the IDE. The SEM image in Figure 2. (D) shows the gold nanostructures of the AuNP modification. At higher magnification (inset) a thin layer can be seen around the Au nanostructures, indicated by the arrows, which can be attributed to the chitosan. This suggests that using $50 \mathrm{ppm} \mathrm{Au}$ in a chitosan solution did not cause additional deposition of AuNPs, but facilitated polymer deposition. Optical and SEM images for an IDE modified with Cht-Au only (i.e., with the AuNP layer suppressed) are presented in Figure S3. (B). The optical image shows a green coloured layer, indicative of chitosan, around the bands in the sensor IDE while under high-resolution SEM, a thin layer, similar to the inset of Figure 2. (D), at the electrode edges, can be observed. EDX analysis of IDEs electrodes modified with AuNPs and AuNPs/Cht-Au modified was undertaken as it is well known that chitosan is rich in amine functional groups. Consequently, EDX can be used to confirm the presence of nitrogen on the surface of a modified electrode. Nitrogen was not present on the electrode modified with AuNPs only (negative values in the table; inset), Figure 2. (E), while it was present on the electrode modified with AuNPs/CHI-Au, (positive $3.43 \%$ in the table, inset), Figure 2. (F). 
A
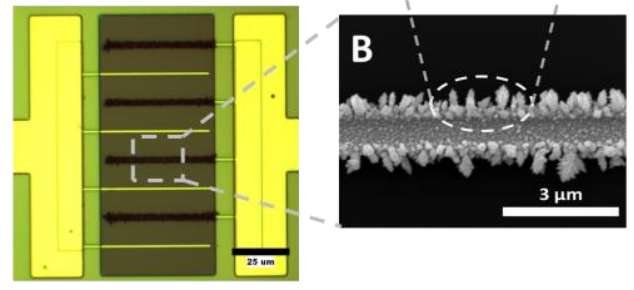

E

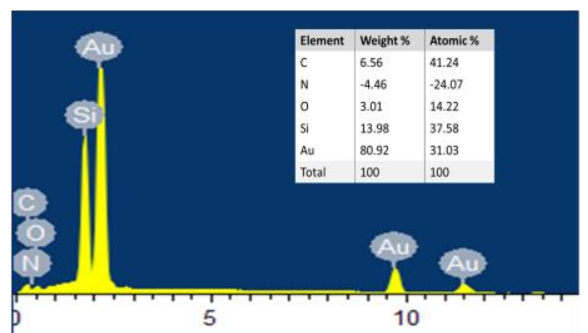

C

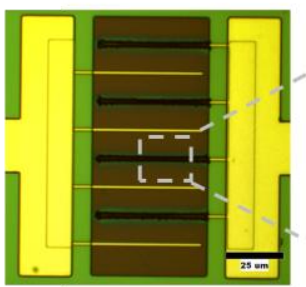

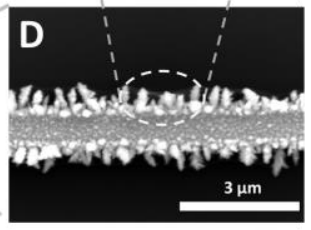

$\mathbf{F}$

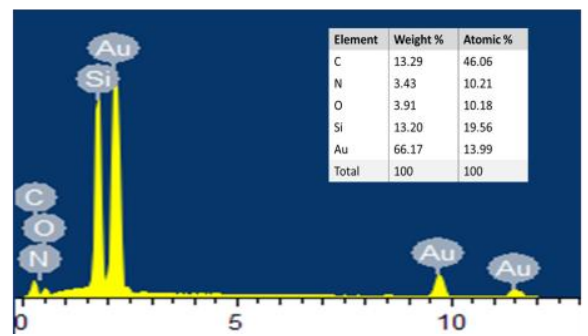

Figure 2 (A) optical image of the AuNPs modified IDE. (B) SEM image of the AuNPs surface. (C) optical image of the AuNPs/Cht-Au modified IDE. (D) SEM image of the AuNPs/Cht-Au surface. (E) EDX spectrum of the AuNPs modified IDE. (F) EDX spectrum of the AuNPs/Cht-Au modified IDE.

\subsection{Confirmation of sensor functionality using fluores- cence}

Following EDX confirmation of the presence of nitrogen at an AuNPs/Cht-Au modified sensor IDE surface, Atto565 NHS ester (a fluorescent dye modified to bind to primary amine groups) was used to confirm the presence of amine groups on the AuNPs/Cht$\mathrm{Au}$ modified IDE and visualise their distribution along the electrode; similar to Wu, Yi [37]. Subsequently, Atto565 modified DNA complementary and non-complementary strands were used to visualise DNA binding to probe ssDNA.

Figure 3. (A) presents a fluorescent image of AuNPs/Cht-Au IDE following $30 \mathrm{~min}$ incubation with Atto565 NHS ester. The observed uniform fluorescent signal strongly suggests that the amine groups are equally distributed along the whole length of the modified sensor IDE. In addition, the high intensity suggests the amine groups are quite dense. By contrast, the lack of fluorescence from the unmodified accumulator IDE confirms the selective deposition of the polymer matrix at the sensor IDE only. Figure 3. (B) presents a fluorescent image of an AuNPs/Cht-Au modified IDE in the absence of Atto565 NHS ester dye confirming that the chitosan layer is not autofluorescent and the fluorescence in Figure 3. (A) was attributed to the functionalised amine groups. Similarly, fluorescence experiments were performed at $\mathrm{Cht}-\mathrm{Au}$ and $\mathrm{Cht}$ only modified IDEs and incubated in Atto565 for 30 mins. Fluorescence was not observed a Cht only modified electrode indicating that chitosan did not deposit onto a pristine gold electrode see Figure S4. (A). By contrast, a uniform fluorescent signal was observed for the ChtAu modified electrode, see Figure S4. (B), however, the signal intensity was significantly reduced compared to electrodes with an underlying AuNPs layer presented in Figure 3. (A). This suggests that the underlying AuNPs layer, below the Cht-Au layer, is essential not only to increase the conductivity of the chitosan film but also to increase the concentration of amine groups.

Subsequently, ssDNA was immobilised at AuNPs/Cht-Au IDEs using $0.2 \%$ glutaraldehyde as a linker molecule. Atto565 modified target (10 nM) DNA was introduced onto an electrode and incubated for 30 minutes. Following hybridisation, unattached DNA was washed away and fluorescent microscopy was used to confirm specific hybridisation of the complementary DNA occurred. Figure 3 (C) presents a fluorescent image following incubation with complementary DNA strands. The observed fluorescence exhibited high intensity and was uniformly distributed across the electrode confirming successful hybridisation. Figure 3 (D) presents a fluorescent image following incubation with Atto565 modified noncomplementary DNA strands. The lack of a fluorescent signal indicates that non-specific binding did not occur.

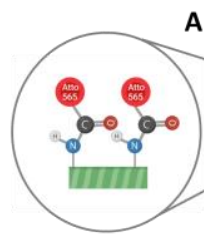

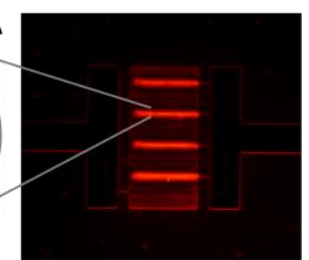

AuNPs/CHI-Au + Atto565

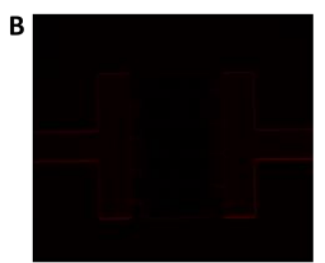

AuNPs/CHI-Au
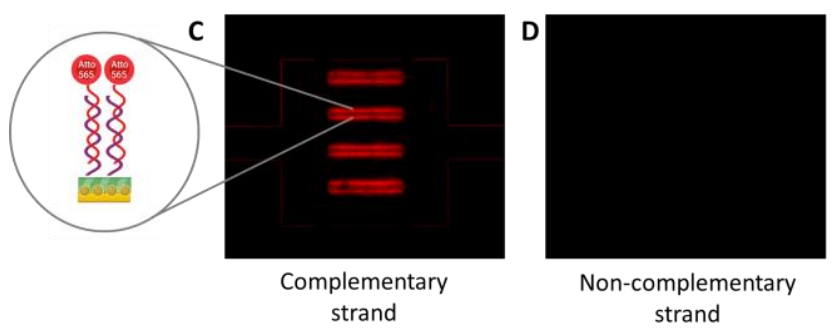

Non-complementary strand

Figure 3 (A) Image under the fluorescent microscope showing the AuNPs/Cht-Au modified sensor IDE after incubation with atto565 NHS ester dissolved in 0.01 M PBS. (B) Image under the fluorescent microscope showing AuNPs/Cht-Au modified sensor IDE 
without the fluorescent dye - negative sample. (C) Image under fluorescent microscopy showing an AuNPSs/CHI-Au/ssDNA modified sensor IDE after incubation with $10 \mathrm{nM}$ DNA strand complementary DNA strand, tagged with atto565 (D) Image under the fluorescent microscopy showing AuNPSs/CHI-Au/ssDNA modified sensor IDE after incubation with $10 \mathrm{nM}$ DNA strand non-complementary to stx 1 gene tagged with atto565.

\subsection{Electrochemical-based DNA detection using meth- ylene blue}

Methylene blue (MB), a redox-active molecule, is known to interact with DNA and the difference in its affinity towards ssDNA and dsDNA has been commonly exploited in the development of electrochemical biosensors [38-40]. The molecule can (i) bind directly to guanine bases [41], (ii) is electrostatically attracted to the negatively charged phosphate backbone of DNA [42], or (iii) intercalate between dsDNA [43, 44]. The mechanisms at which it will interact with DNA can be influenced by the experimental parameters used. In this work, we focused on enhancing MB intercalation between dsDNA and detecting DNA hybridisation based on an increased SWV signal compared to ssDNA. One parameter that was shown to affect MB to dsDNA binding efficiency is the ionic strength of the solution [42]. Kara, Kerman [45] have suggested that the concentration of $\mathrm{Cl}^{-}$ions can influence the efficiency of $\mathrm{MB}$ intercalation between dsDNA. In their work, the MB intercalation between dsDNA was preferred over the other type of MB interactions at 10 $\mathrm{mM} \mathrm{NaCl}$. In this work, DNA detection was undertaken using 50 $\mu \mathrm{M}$ MB dissolved in $0.02 \mathrm{M}$ HEPES buffer with $10 \mathrm{mM} \mathrm{KCl}$ and an accumulation step to achieve the highest efficiency of intercalation.

The electrochemical detection of DNA in this work was undertaken by accumulating MB at a DNA modified electrode followed by its reduction to leukomethylene blue recorded with SWV. An OCP [46] commonly used for the accumulation of methylene blue in DNA sensor development [45] was applied to an accumulator electrode as described above. Figure 4. (A) and Figure 4. (B) show square wave voltammograms before and after exposure to $50 \mathrm{nM}$ of complementary DNA, with and without applying OCP to the accumulator IDE, respectively. In both cases, the accumulation was done by incubating the chip for 10 minutes in $50 \mu \mathrm{M}$ MB dissolved in $0.02 \mathrm{M}$ HEPES and $0.01 \mathrm{M} \mathrm{KCl}(\mathrm{pH} 7)$.

The peak increase for dsDNA $(50 \mathrm{nM})$ over ssDNA, without applying an OCP, was $2.28 \mathrm{nA}$ equivalent to $\sim 50 \%$ increase in the measured signal. When an OCP was applied to an accumulator IDE, it was observed that the ssDNA peak current had a similar magnitude compared to the static accumulation. By contrast, a significant increase in peak current on the binding of complementary dsDNA $(50 \mathrm{nM})$ was observed with a $\Delta$ peak height current (between ssDNA and dsDNA) increased to $5.05 \mathrm{nA}$, over a $100 \%$ increase. This suggests that applying an OCP $(8 \mathrm{mV}$, see Figure S5.) increases the efficiency of MB intercalation between dsDNA without affecting its interaction with ssDNA.

In Figure 1 (D) we have previously shown that electrodeposition of AuNPs as a first layer significantly increased the conductivity of the modified sensor IDE. We further evaluated how AuNPs affected DNA detection using MB. Figure 4 (C) summarises peak current height before (orange) and after (green) hybridisation with complementary strand when the electrode was not modified and modified (400 ppm) with the first layer of AuNPs. It was found that the difference between ssDNA and dsDNA was hard to distinguish without the layer of AuNPs. This is in agreement with Figure 1 (D) where the conductivity of the electrode decreased after Cht-Au modification. However, in the presence of the underlying AuNPs layer, a significant increase in MB measured current was observed between ssDNA and dsDNA, again confirming the need for this layer.
Finally, the effect of ssDNA concentration immobilised at AuNPs/Cht-Au modified sensors IDE on the MB reduction signal was assessed. Figure S6 presents the peak height increase observed after dsDNA ( $50 \mathrm{nM})$ binding with different concentrations of immobilised ssDNA (i) $0.25 \mu \mathrm{M}$, (ii) $0.5 \mu \mathrm{M}$, (iii) $1 \mu \mathrm{M}$ and (iv) 1.5 $\mu \mathrm{M}$, respectively. The best response was achieved when $0.5 \mu \mathrm{M}$ probe concentration was used. This suggests that with $0.25 \mu \mathrm{M}$ there was not enough probe ssDNA attached to the surface, while at higher concentrations $1 \mu \mathrm{M}$ and $1.5 \mu \mathrm{M}$ the probe density was too high to achieve optimal hybridisation due to steric hindrance. Figure 5. (D) presents square wave voltammograms after incubation of a sensor with $50 \mathrm{nM}$ of (i) target DNA, (ii) non-complementary strand and (iii) a DNA strand containing 3 base mismatches. An increase in current magnitude was not observed for the noncomplementary or mismatched strands. However, a significant increase was observed for the complementary strand, confirming the specificity of the developed biosensor.
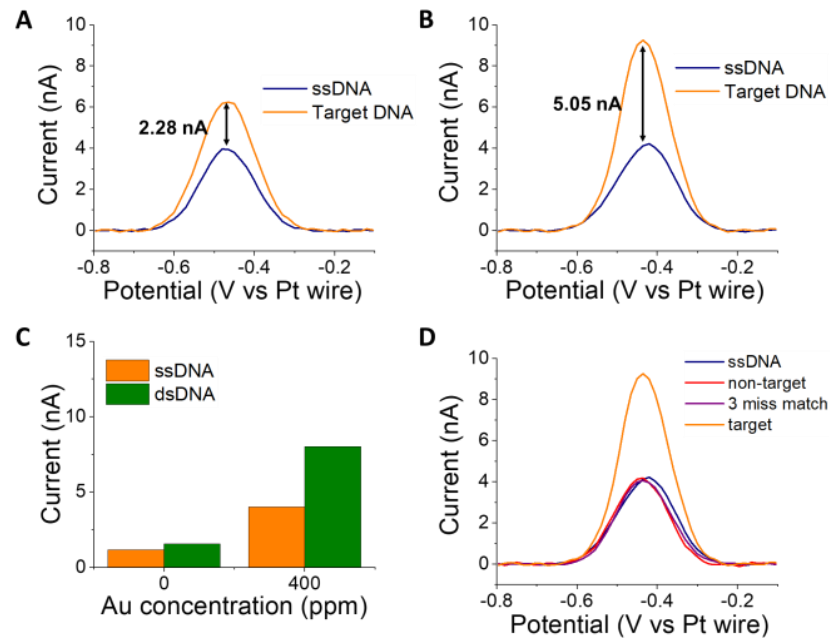

Figure 4. (A) Square wave voltammograms before and after exposure to $50 \mathrm{nM}$ complementary DNA strand after $10 \mathrm{~min}$ of static accumulation (no OCP) at $50 \mu \mathrm{M}$ MB in $0.02 \mathrm{M}$ HEPES and 0.01 $\mathrm{M} \mathrm{KCl}, \mathrm{pH}$ 7. (B) Square wave voltammograms before and after exposure to $50 \mathrm{nM}$ complementary DNA strand when OCP was applied to accumulator IDE for 5 minutes followed by 5 minutes of a static accumulation. (C) Peak current heights before and after exposure to $50 \mathrm{nM}$ complementary DNA strand at sensor IDE modified with Cht-Au layer deposited for $20 \mathrm{~s}$ with (i) no underlying AuNPs and (ii) underlying AuNPs. (D) Square wave voltammograms before and after exposure to $50 \mathrm{nM}$ (i) complementary DNA strand, (ii) non-complementary strand, (iii) 3 bases miss-matched strand.

To find the limit of detection and establish a calibration curve, the AuNPs/Cht-Au/ssDNA modified IDEs were incubated for 20 minutes with a series of DNA concentrations diluted with $0.1 \mathrm{M}$ PBS buffer. The peak height was found to increase linearly between $100 \mathrm{aM}$ and $1 \mu \mathrm{M}$ of target DNA. Figure 5. (A) shows the calibration curve where the difference between dsDNA and ssDNA peak height current was plotted against the logarithm of DNA concentration. All data points represent an average value of four measurements (from different sensors) and standard deviation for each point. The calibration line showed excellent linearity, with a correlation coefficient of $\mathrm{R}^{2}=0.995$. The lowest measured concertation, and thus the limit of detection, was observed to be $100 \mathrm{aM}$. Figure 5. (B) presents square wave voltammograms for each concentration. The baseline was normalised to allow better visualisation

To evaluate the specificity of the developed sensor in complex samples, chromosomal DNA extracted from different strains of $E$. coli, viz, stxl positive (O103 and O157) and stxl negative (O91 and 
12900) and non-E. coli (Listeria monocytogenes and Bacillus cereus) was tested. After the extraction, the DNA was diluted 5 times with the PBS buffer, heated to $95^{\circ} \mathrm{C}$ for 5 mins and immediately cooled on ice for $60 \mathrm{~s}$. The samples were then incubated on a sensor for 30 minutes. Figure 5. (C) presents combined square wave voltammograms from different sensors following incubation with the chromosomal DNA. It can be seen that only DNA extracted from E. coli containing the stxl gene (O103 and O157) resulted in an increase in the signal, further demonstrating the selectivity of the sensor to complex samples. The signal from the 0103 strain was higher than O157, suggesting that it either contained more stxl gene or it was more readily available to hybridise with the sensor. Figure 5. (D) summarises the difference in peak height current after detection using chromosomal DNA samples (Figure 5. (C)). Each data point represents the average value of three measurements from different sensors.
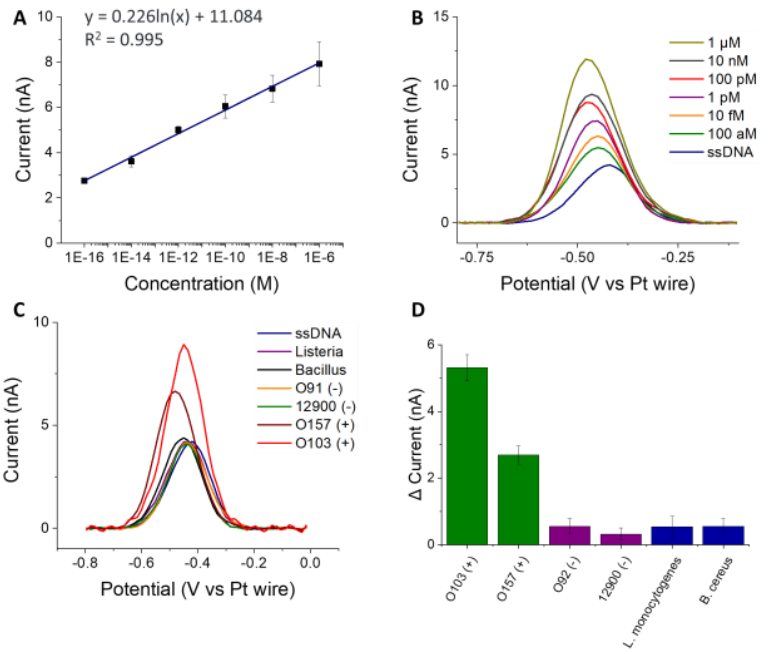

Figure 5 (A) Calibration line representing the difference between peak height current of dsDNA and ssDNA plotted against the logarithm of the target DNA concentration. (B) Square wave voltammograms corresponding to each concentration of the calibration curve. SWV parameters were frequency $75 \mathrm{~Hz}$, pulse amplitude 75 $\mathrm{mV}$. The baseline was corrected for a better visual analysis of the voltammograms (C) Square wave voltammograms for detection of the chromosomal DNA. The baseline was corrected to enable a better visual analysis of the voltammograms. (D) $\Delta$ peak current heigh between MB response at ssDNA and after incubation with chromosomal DNA.

\section{Conclusions}

In this work, we presented a highly sensitive, on-chip, DNA-based sensor for detection of stxl gene from STEC, using gold IDEs enabled by the pre-concertation of MB at the sensor. In this work, we developed a facile method for the specific modification of the sensor IDE with Cht-Au nanocomposite, without affecting the accumulator IDE. This was confirmed using fluorescent techniques. Following this, we demonstrated for the first time that applying OCP to the accumulator IDE significantly improved MB intercalation between dsDNA and therefore allowed a highly sensitive detection. Finally, we established a calibration curve between $100 \mathrm{aM}$ to $1 \mu \mathrm{M}$ of synthetic target strand and specifically detected chromosomal DNA extracted from overnight bacterial cultures. This work is highly relevant for the development of novel point-of-use devices for rapid pathogens detection and can be further extended to allow multiplex detection in the future by modifying separate electrodes with different probe sequences. In addition, it demonstrates a novel use of the IDE where one comb can be specifically modified with a biorecognition molecule while the other comb can be used for other applications such as molecule accumulation or electrochemical pH control.

\section{ASSOCIATED CONTENT}

\section{Supporting Information:}

"Supporting information: Additional figures regarding sensor optimization mentioned in text including photographs and electrochemical characterisation."

This material is available free of charge via the Internet at http://pubs.acs.org.

\section{AUTHOR INFORMATION}

\section{Corresponding Author}

alan.oriordan@tyndall.ie

\section{Author Contributions}

The manuscript was written through contributions of all authors. / All authors have given approval to the final version of the manuscript.

Notes

The authors declare no competing financial interests.

\section{ACKNOWLEDGMENT}

This study was funded in part by the Teagasc Walsh Scholarship Scheme (Ref: 2016024) and has emanated in part from research conducted with the financial support of Science Foundation Ireland (SFI) and the Department of Agriculture, Food and Marine on behalf of the Government of Ireland under Grant Number [16/RC/3835].

\section{REFERENCES}

1. McCarthy, S.C., et al., An Overview of Shiga-Toxin Producing Escherichia coli Carriage and Prevalence in the Ovine Meat Production Chain. Foodborne Pathogens and Disease, 2021. 18(3): p. 147-168.

2. Duffy, G., C.M. Burgess, and D.J. Bolton, A review of factors that affect transmission and survival of verocytotoxigenic Escherichia coli in the European farm to fork beef chain. Meat science, 2014. 97(3): p. 375383.

3. McCabe, E., et al., An investigation of shedding and super-shedding of Shiga toxigenic Escherichia coli O157 and E. coli O26 in cattle presented for slaughter in the Republic of Ireland. Zoonoses and public health, 2019. 66(1): p. 83-91.

4. Beutin, L. and A. Martin, Outbreak of Shiga toxin-producing Escherichia coli (STEC) O104: H4 infection in Germany causes a paradigm shift with regard to human pathogenicity of STEC strains. Journal of food protection, 2012. 75(2): p. 408-418.

5. Panel, E.B., et al., Pathogenicity assessment of Shiga toxin-producing Escherichia coli (STEC) and the public health risk posed by contamination of food with STEC. EFSA Journal, 2020. 18(1): p. e05967.

6. Kralik, P. and M. Ricchi, A basic guide to real time PCR in microbial diagnostics: definitions, parameters, and everything. Frontiers in microbiology, 2017. 8: p. 108 .

7. Tavallaie, R., et al., Nucleic acid hybridization on an electrically reconfigurable network of gold-coated magnetic nanoparticles enables microRNA detection in blood. Nature nanotechnology, 2018. 13(11): p. 10661071 .

8. Gupta, N., et al., Point-of-Care PCR Assays for COVID-19 Detection. Biosensors, 2021. 11(5): p. 141.

9. Ali, M., et al., Development of an advanced DNA biosensor for pathogenic Vibrio cholerae detection in real sample. Biosensors and Bioelectronics, 2021. 188: p. 113338.

10. Liu, A., et al., Development of electrochemical DNA biosensors. TrAC Trends in Analytical Chemistry, 2012. 37: p. 101-111.

11. Lawal, A.T., Progress in utilisation of graphene for electrochemical biosensors. Biosensors and Bioelectronics, 2018. 106: p. 149-178. 
12. Rashid, J.I.A. and N.A. Yusof, The strategies of DNA immobilization and hybridization detection mechanism in the construction of electrochemical DNA sensor: A review. Sensing and bio-sensing research, 2017. 16: p. 19-31.

13. Faria, H.A.M. and V. Zucolotto, Label-free electrochemical DNA biosensor for zika virus identification. Biosensors and Bioelectronics, 2019. 131: p. 149-155.

14. Mazlan, N., et al. Interdigitated electrodes as impedance and capacitance biosensors: A review. in AIP Conference proceedings. 2017. AIP Publishing LLC.

15. Seymour, I., et al., Electrochemical detection of free-chlorine in Water samples facilitated by in-situ $\mathrm{pH}$ control using interdigitated microelectrodes. Sensors and Actuators B: Chemical, 2020. 325: p. 128774.

16. Daly, R., et al., Platinum-Based Interdigitated Micro-Electrode Arrays for Reagent-Free Detection of Copper. Sensors, 2021. 21(10): p. 3544.

17. Wang, L., et al., A sensitive DNA capacitive biosensor using interdigitated electrodes. Biosensors and Bioelectronics, 2017. 87: p. 646653.

18. Parmin, N.A., et al., Voltammetric determination of human papillomavirus 16 DNA by using interdigitated electrodes modified with titanium dioxide nanoparticles. Microchimica Acta, 2019. 186(6): p. 336.

19. Odeh, A.A., et al., A needle-like $\mathrm{Cu} 2 \mathrm{CdSnS} 4$ alloy nanostructure-based integrated electrochemical biosensor for detecting the DNA of Dengue serotype 2. Microchimica Acta, 2017. 184(7): p. 2211-2218.

20. Zhang, J., et al., Rapid detection of Escherichia coli based on 16S rDNA nanogap network electrochemical biosensor. Biosensors and Bioelectronics, 2018. 118: p. 9-15.

21. Singh, A., et al., Graphene oxide-chitosan nanocomposite based electrochemical DNA biosensor for detection of typhoid. Sensors and Actuators B: Chemical, 2013. 185: p. 675-684.

22. Ulianas, A., et al., An electrochemical DNA microbiosensor based on succinimide-modified acrylic microspheres. Sensors, 2012. 12(5): p. 5445-5460.

23. Geng, P., et al., A DNA sequence-specific electrochemical biosensor based on alginic acid-coated cobalt magnetic beads for the detection of E. coli. Biosensors and Bioelectronics, 2011. 26(7): p. 3325-3330.

24. Jiang, Y. and J. Wu, Recent development in chitosan nanocomposites for surface-based biosensor applications. Electrophoresis, 2019. 40(16-17): p. 2084-2097.

25. Tiwari, I., et al., Electrochemical detection of a pathogenic Escherichia coli specific DNA sequence based on a graphene oxide-chitosan composite decorated with nickel ferrite nanoparticles. RSC advances, 2015. 5(82): p. 67115-67124.

26. Teixeira, S., et al., Chitosan/AuNPs modified graphene electrochemical sensor for label-free human chorionic gonadotropin detection. Electroanalysis, 2014. 26(12): p. 2591-2598.

27. Juska, V.B. and M.E. Pemble, A dual-enzyme, micro-band array biosensor based on the electrodeposition of carbon nanotubes embedded in chitosan and nanostructured $\mathrm{Au}$-foams on microfabricated gold band electrodes. Analyst, 2020. 145(2): p. 402-414.

28. Tabasi, A., A. Noorbakhsh, and E. Sharifi, Reduced graphene oxide-chitosan-aptamer interface as new platform for ultrasensitive detection of human epidermal growth factor receptor 2. Biosensors and Bioelectronics, 2017. 95: p. 117-123.

29. Tian, L., et al., A facile DNA strand displacement reaction sensing strategy of electrochemical biosensor based on N-carboxymethyl chitosan/molybdenum carbide nanocomposite for microRNA-21 detection. Biosensors and Bioelectronics, 2018. 122: p. 43-50.
30. Cui, H.-F., et al., A highly stable acetylcholinesterase biosensor based on chitosan-TiO2-graphene nanocomposites for detection of organophosphate pesticides. Biosensors and Bioelectronics, 2018. 99: p. 223-229.

31. Yadav, A.K., et al., A highly sensitive label-free amperometric biosensor for norfloxacin detection based on chitosan-yttria nanocomposite. International journal of biological macromolecules, 2020. 151: p. 566575 .

32. Du, Y., et al., A simple method to fabricate a chitosan-gold nanoparticles film and its application in glucose biosensor. Bioelectrochemistry, 2007. 70(2): p. 342-347.

33. Wahl, A., et al., Electroanalysis at ultramicro and nanoscale electrodes: a comparative study. Journal of The Electrochemical Society, 2013. 161(2): p. B3055.

34. Wasiewska, L.A., et al., Reagent free electrochemical-based detection of silver ions at interdigitated microelectrodes using in-situ $\mathrm{pH}$ control. Sensors and Actuators B: Chemical, 2021. 333: p. 129531.

35. Xu, S., et al., Electrochemical DNA biosensor based on graphene oxide-chitosan hybrid nanocomposites for detection of Escherichia coli O157: H7. Int. J. Electrochem. Sci, 2017. 12: p. 3443-3458.

36. Yang, Y., et al., Enhanced charge transfer by gold nanoparticle at DNA modified electrode and its application to label-free DNA detection. ACS applied materials \& interfaces, 2014. 6(10): p. 7579-7584.

37. Wu, L.-Q., et al., Spatially selective deposition of a reactive polysaccharide layer onto a patterned template. Langmuir, 2003. 19(3): p. 519 524.

38. Hassan, R.A., L.Y. Heng, and L.L. Tan, Novel DNA biosensor for direct determination of carrageenan. Scientific reports, 2019. 9(1): p. 19.

39. Zhao, L.-L., et al., Ultrasensitive detection of microRNA based on a homogeneous label-free electrochemical platform using G-triplex/methylene blue as a signal generator. Analytica chimica acta, 2020. 1116: p. 62-69.

40. Pothipor, C., et al., A highly sensitive electrochemical microRNA-21 biosensor based on intercalating methylene blue signal amplification and a highly dispersed gold nanoparticles/graphene/polypyrrole composite. Analyst, 2021. 146(8): p. 2679-2688.

41. Yang, W., et al., Evidence for the direct interaction between methylene blue and guanine bases using DNA-modified carbon paste electrodes. Electroanalysis: An International Journal Devoted to Fundamental and Practical Aspects of Electroanalysis, 2002. 14(18): p. 1299-1302.

42. Farjami, E., et al., DNA interactions with a methylene blue redox indicator depend on the DNA length and are sequence specific. Analyst, 2010. 135(6): p. 1443-1448.

43. Rohs, R., et al., Methylene blue binding to DNA with alternating GC base sequence: a modeling study. Journal of the American Chemical Society, 2000. 122(12): p. 2860-2866.

44. Vardevanyan, P., et al., Mechanisms for binding between methylene blue and DNA. Journal of Applied Spectroscopy, 2013. 80(4): p. 595 599.

45. Kara, P., et al., Electrochemical genosensor for the detection of interaction between methylene blue and DNA. Electrochemistry Communications, 2002. 4(9): p. 705-709.

46. Munoz, A.I., N. Espallargas, and S. Mischler, Tribocorrosion: Definitions and Relevance, in Tribocorrosion. 2020, Springer. p. 1-6. 
For Table of Contents Only (created with biorender.com)

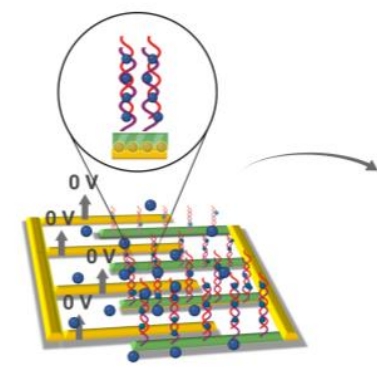

$\mathrm{MB}$ intercalation at $\mathrm{OCP}$

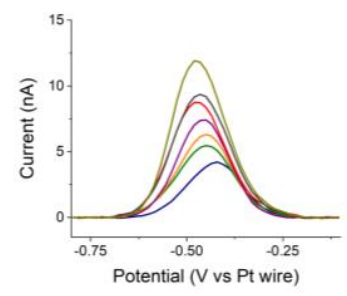

Detection using SWV 